\title{
RURALIZAÇÃO DA LUTZOMYIA INTERMEDIA, UM PROVÁVEL CASO DE PRE-ADAPTAÇĀO
}

Lais Clark Lima*

LIMA, L.C. Ruralização da Lutzomyia intermedia, um provável caso de pré-adaptação.

Rev. Saúde públ., S. P.aulo, 20:102-4, 1986.

RESUMO: A Lutzomyia intermedia há muito vem sendo encontrada em áreas de colonização antiga. Analisando dados de capturas deste flebotomíneo, com diferentes iscas e em diferentes locais, acredita-se que esta espécie está pré-adaptada a ambientes abertos e a se alimentar em mamíferos, entre eles o homem.

UNITERMOS: Leishmaniose tegumentar. Lutzomyia intermedia. Ecologia.

A leishmaniose tegumentar americana, que vinha sendo considerada uma endemia essencialmente florestal (Pessôa e Barretto ${ }^{13}$, 1948), vem ocorrendo em áreas rurais de colonização antiga (Souza e col. ${ }^{14}$, 1981; Lima e col. ${ }^{11}$, 1981) e esporadicamente em áreas periurbanas (Aragão ${ }^{1}, 1922$ ). Este fato foi há muito registrado, porém não comentado por Barretto ${ }^{4}$ (1943), que em uma tabela desse trabalho mostra que a Lutzomyia intermedia - responsável pela transmissão desta leishmaniose (Aragão ${ }^{1}, 1922$ ) era muito mais abundante nas áreas de colonização antiga, do Estado de São Paulo, do que naquelas onde ainda predominavam as florestas. Forattini ${ }^{5,6}$ $(1953,1960)$ foi quem primeiro observou a tendência rural desta espécie de flebotomíneo, ao relatar a dominância da mesma em ambiente alterado pelo homem.

O encontro da $L$. intermedia, em domicílios (Forattini, 5 1953; Araújo $F^{\mathbf{0} 3}$, 1978; Lima e col.11, 1981), peridomicílios (Forattini 5,b, 1953, 1960; Fundação Oswaldo Cruz (FIOCRUZ), 1974**; Souza e col. ${ }^{14}$, 1981) e em campo aberto
(Gomes e col. ${ }^{7,8,9,10}, 1978,1980,1982$, 1983) faz com que se medite sobre o comportamento da espécie.

Senão vejamos, Gomes e col. ${ }^{\rtimes}$ (1980) em capturas de flebotomíneos em galinheiros experimentais, realizadas no $\mathrm{Va}$ le do Ribeira, Estado de São Paulo, obtiveram alta densidade de $L$. intermedia na margem da mata, seguida do ambiente extraflorestal e densidade nula dentro da mata (Tabela) - fato também observado por Souza *** em Jacarepaguá, Rio de Janeiro. Todavia, Gomes e col. ${ }^{10}$ (1983) tiveram êxito nas capturas de L. intermedia com isca humana dentro da mata, na mesma área do Vale do Ribeira. Além disso, Lima e col. ${ }^{12}$ (1982), ao efetuarem capturas de flebotomíneos, em Jacarepaguá, Rio de Janeiro, obtiveram alto rendimento, da mencionada espécie, nos chiqueiros - fato há muito observado por Forattini ${ }^{5}$ (1953) - e pouco significante nos galinheiros. E ainda foi notável a quantidade de $L$. intermedia encontrada por Lima e col.11 (1981) e pela FIOCRUZ** em abrigos de muares em Campo Grande e Jacarepaguá, respectivamente, no Estado do Rio de Janeiro.

\footnotetext{
- Do Departamento de Ciências Biológicas da Escola Nacional de Saúde Pública - FIOCRUZ - Caixa Postal 926 - 21.040 - Rio de Janeiro, RJ - Brasil.

** Documento interno.

** Comunicação pessoal.
} 
LIMA, L.C. Ruralização da Lutzomyia intermedia, um provável caso de pré-adaptação. Rev. Saúde públ., S, Paulo, 20:102-4, 1986.

A Tabela mostra estes fatos, eluci- mamíferos - no caso, muar, porco e dando a preferência da $L$. intermedia por homem.

TABELA

Lutzomyia intermedia capturadas em diferentes locais e iscas.

\begin{tabular}{|c|c|c|c|c|c|c|c|}
\hline Isca & $\begin{array}{c}\text { Isca } \\
\text { humana }\end{array}$ & $\begin{array}{l}\text { Gali- } \\
\text { nheiro }\end{array}$ & $\begin{array}{l}\text { Gali- } \\
\text { nheiro }\end{array}$ & $\begin{array}{l}\text { Gali- } \\
\text { nheiro }\end{array}$ & $\begin{array}{c}\text { Gali- } \\
\text { nheiro }\end{array}$ & $\begin{array}{c}\text { Abrigo de } \\
\text { porcos }\end{array}$ & $\begin{array}{l}\text { Abrigo } \\
\text { de muar }\end{array}$ \\
\hline Localização & $\begin{array}{c}\text { Interior } \\
\text { da floresta }\end{array}$ & $\begin{array}{l}\text { Interior } \\
\text { da floresta }\end{array}$ & $\begin{array}{l}\text { Orla da } \\
\text { floresta }\end{array}$ & $\begin{array}{c}\text { Campo } \\
\text { aberto }\end{array}$ & $\begin{array}{l}\text { Perido- } \\
\text { micílio }\end{array}$ & $\begin{array}{l}\text { Perido- } \\
\text { micílio }\end{array}$ & $\begin{array}{l}\text { Perido- } \\
\text { micílio }\end{array}$ \\
\hline Número & 886 & - & 109.147 & 24.707 & 74 & 793 & 294 \\
\hline $\begin{array}{l}\text { Horas-homem } \\
\text { trabalhadas }\end{array}$ & 1.152 & 85 & 1.150 & 451,5 & 4.4 & 8,3 & 3,1 \\
\hline $\begin{array}{l}\text { Rendimento/ } \\
\text { homem-hora }\end{array}$ & 0,8 & - & 94,9 & 54,7 & 16,8 & 95,5 & 94,8 \\
\hline Percentual & 0,2 & - & 26,5 & 15,3 & 4,7 & 26,7 & 26,5 \\
\hline
\end{tabular}

Fonte: Gomes e col. ${ }^{R}{ }^{10}$ (1980, 1983); Lima e col. ${ }^{11}$ (1981); Lima (dados inéditos)

Assim sendo, estes dados justificam a afirmação de Gomes e col. ${ }^{8}$ (1980) quando sugerem que a $L$. intermedia "esteja adaptada ao efeito marginal". Ora, o comportamento deste flebotomíneo está de acordo com o exemplo de Vanzolini e Williams ${ }^{15}(1981)$, ao reportarem que a probabilidade de lagartos de floresta se adaptarem à semi-aridez seria maior se a população estivesse pré-adaptada, ou seja, fosse tolerante às duas ecologias, preferindo as margens entre elas. $E$ ainda está de acordo com a constatação de Aragão " (1983), que a colonização de biótopos artificiais - incluindo moradia de boa qualidade - por triatomíneos está sempre associada à presença de animais por eles preferidos na natureza, indicando que o "fato de umas espécies ocorrerem em domicílios e outras em abrigos de aves é decorrência de préadaptação".

A L. intermedia tem, portanto, uma pré-adaptação a dois elementos essenciais na existência de uma espécie: o alimento e o ambiente no qual precisa circular para exercer suas funções vitais.

\section{AGRADECIMENTO}

Ao Dr. Mario B. Aragão, pela sugestão do tema.

LIMA. L.C. [The ruralization of Lutzomyia intermedia, a possible casé of preadaptation].

ABSTRACT: Lutzomyia intermedia has for a long time been found in areas which have long time been settled. Data on sandflies caught with different baits at different places led to believe that this species is preadapted to open environments and to feeding on mamals, including man.

UNITERMS: Leishmaniasis, micocutaneous, Lutzomyia intermedia. Ecology.

\section{REFERENCIAS BIBLIOGRÁFICAS}

1. ARAGÃO, M.B. Transmissão da leishmaniose no Brasil pelo Phlebotomus intermedius. Brasil-med., Rio de Janeiro, 36: 129-30, 1922.
2. ARAGÃO, M.B. Domiciliação de triatomíneos ou pré-adaptação à antropofilia e à ornitofilia? Rev. Saúde públ., São Paulo, 17:51-5, 1983. 
LIMA, L.C. Ruralização da Lutzomyia intermedia, um provável caso de pré-adaptação. Rev. Saúde públ., S. Paulo, 20:102-4, 1986.

3. ARAÚJO F. ${ }^{\circ}$, N.A. Epidemiologia de leishmaniose tegumentar americana na Ilha Grande, Rio de Janeiro. Esutdos sobre a infecção humana, reservatórios e transmissores. Rio de Janeiro, 1978. [Dissertação de Mestrado UFRJ]

4. BARRETTO, M.P. Observações sobre a biologia, em condições naturais, dos flebótomos do Estado de São Paulo (Diptera, Psychodidae). São Paulo, 1943. [Tese Livre Doncêcia - Faculdade de Medicina de Ribeirão Preto da USP]

5. FORATTINI, O. P. Notas sobre criadouros naturais de flebótomos em dependências peridomiciliares, no Estado de São Paulo. Arq. Fac. Hig. Saúde públ., S. Paulo, 7:157-65, 1953.

6. FORATTINI, O.P. Novas observações sobre a biologia de flebótomos em condições naturais (Diptera, Psychodidae). Arq. Hig. Saúde públ., S. Paulo, 25:209. $15,1960$.

7. GOMES, A.C. et al. Flebotomíneos encontrados em galinheiros experimentais nos Estados de São Paulo e Minas Gerais (Brasil) e algumas observações ecológicas. Rev. Saúde públ., S. Paulo, 12:403-7, 1978.

8. GOMES, A.C. et al. Aspectos ecológicos da leishmaniose tegumentar americana, 1. Estudo experimental da freqüência de flebotomíneo e ecótopos artificiais com referência especial a Psychodopygus intermedius. Rev. Saúde públ., S. Paulo, 14:540-56, 1980.

9. GOMES, A.C. et al. Aspectos ecológicos da leishmaniose tegumentar americana. 2. Ecótopo artificial como abrigo de Psychodopygus intermedius e observações sobre alimentação e reprodução sob influência de fatores físicos naturais. Rev. Saúde públ., S. Paulo, 16:149-59, 1982.

10. GOMES, A.C. et al. Aspectos ecológicos da leishmaniose tegumentar americana. 3. Observações naturais sobre o ritmo diário de Psychodopygus intermedius em ambiente florestal e extra-florestal. Rev. Saúde publ. S. Paulo, 17:23-30, 1983.

11. LIMA, L.C.R. et al. Flebotomíneos em áreas de ocorrência de leishmaniose tegumentar no bairro de Campo Grande, Rio de Janeiro, Brasil. Rev. bras. Malar., 33:64-74, 1981.

12. LIMA, L.C.R. et al. Aspectos epidemiológicos da leishmaniose tegumentar em área submetida a profilaxia no Rio de Janeiro. In: Congresso da Sociedade Brasileira de Parasitologia, Porto Alegre, 1982. Resumos. Porto Alegre, 1982. p. 160 .

13. PESSOA, S.B. \& BARRETTO, M.P. Leishmaniose tegumentar americana. Rio de Janeiro, Ministério da Educação e Saúde, 1948.

14 SOUZA, M.A. et al. Leishmaniose viscera] no Rio de Janeiro. 1 - Flebotomíneos da área de procedência de caso humano autóctone. Mem. Int. Oswaldo Cruz, Rio de Janeiro, 76:161-8, 1981.

15. VANZOLINI, P.E. \& WILLIAMS, E.E. The vanishing refuge: a mechanism for ecogeographic speciation. Pap avuls. Zool., S. Paulo, 34:251-5, 1981.

Recebido para publicação em 18/07/1985. Reapresentado em 25/11/1985

Aprovado para publicação em 27/11/1985 